\title{
Superconductive Energy Storage for Power Systems
}

\author{
ROGER W. BOOM AND HAROLD A. PETERSON
}

\begin{abstract}
The use of large superconducting inductors for "pumped" energy storage as an alternate to pumped hydrostorage is discussed. It is suggested that large units might be developed at less than $\$ 200 / \mathrm{kW}$ and with losses less than the 50 percent representative of pumped hydrostorage. Particular notice is taken of the ability of such peaking units to damp overall power system electromechanical oscillations. Attention is also given to the stress considerations in magnet windings and to the optimization of superconductor and structural material. A preliminary analysis is made for a $400 \mathrm{MW}-10000 \mathrm{MWh}$ unit.
\end{abstract}

\section{INTRODUCTION}

Energy storage for power systems with superconducting magnets has received relatively little attention. Most of the studies $[1,2,3]$ which have been made deal with pulsed energy storage and show that there are many advantages for superconducting inductors over capacitors. In general charge and discharge times have been short. The two papers which have dealt with long charge and discharge times show that losses are not excessive for superconducting inductors used as storage elements in a power system $[4,5]$.

A "pumped" magnetic storage system connected to a three phase line would consist of a large superconducting inductor, a helium refrigerator and dewar system to keep the temperature well below the critical temperature for the superconductor, and an ac/dc converter. Referring to Fig. 1, the storage inductance to be "charged" is the only load on the rectifier inverter. The voltage $E$ is given by an equation of the form

$$
E=E_{d 0} \cos \alpha-I X=L \frac{d I}{d t}
$$

where $E_{d 0}$ is the voltage across the bridge when the current $I$ is equal to zero and $X$ is the commutating reactance. Clearly, for a given set of constants, the delay angle $\alpha$ (which can be varied from 0 to 180 degrees ideally) is the key to the control of rate of charge, including both positive and negative, the latter corresponding to discharging the inductance $L$. Thus, we have inherently continuous control of the reversible process corresponding to the pumped hydrosystem through-

Manuscript received March 1, 1972; revised May 15, 1972. Paper 59.1 , presented at the 1972 INTERMAG Conference, Kyoto, Japan, April 10-13.

R. W. Boom is with the Departments of Nuclear and Metallurgical Engineering, University of Wisconsin, Madison, Wis.

H. A. Peterson is with the Department of Electrical Engineering, University of Wisconsin, Madison, Wis.

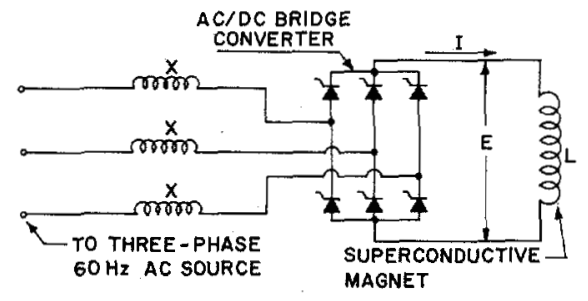

Fig. 1. Basic introductory system.

out the motoring and generating portions of the cycle. For example, if $L$ is 3440 henries and $E$ is held at 8000 volts for $4.3 \times 10^{4}$ seconds or 12 hours, then $4800 \mathrm{MWh}$ can be stored at $10^{5}$ amps at an average rate of $400 \mathrm{MW}$. Note that the thyristor firing angles can be varied so that zero voltage is impressed across the storage inductor. Thus, at full charge the storage current which flows in the inductor, line, and generator can be held constant. Alternatively, the inductor could be shorted with an internal superconducting switch and disconnected from the system when fully charged.

Energy stored in the superconducting magnet is returnable to the ac system under smooth continuous control. Distortions to the voltage waveform of the ac system would result, as is well known. While not the subject of this paper, it should be recognized that converter systems with variable firing angles such as are implied here are essentially no different from those used in modern high voltage direct current (HVDC) power transmission. Appropriately designed filters can be used to reduce harmonics to acceptable levels compatible with quality power standards.

The inefficiencies encountered are those in the conventional ac system, in the converter bridge, and the refrigeration required to balance electrical, magnetic, mechanical, and thermal losses into the cryogenic enclosure. Preliminary loss estimates indicate that, in total, these losses are less than the standard $1 \mathrm{~kW}$ lost for $2 \mathrm{~kW}$ recovered which is usually realized for hydrostorage installations. As an example, let us list the losses for a $10000 \mathrm{MWh}$ unit with a $400 \mathrm{MW}$ reversible rating. For convenience it is assumed that the unit is never discharged to less than $5200 \mathrm{MWh}$. Thus only half of the energy or $400 \mathrm{MW}$ for $12 \mathrm{~h}$ is exchanged with the power system. All losses into the cryogenic enclosure have been translated to an ambient temperature equivalent loss by using a conservative refrigeration factor of 500 watts per watt. As an ambient loss these losses are then quoted below in terms of percent of stored energy or of reversible power, whichever is appropriate. The losses in the bridge and conventional ac system might amount to 2 percent, $8 \mathrm{MW}$ or $192 \mathrm{MWh}$ for a $24 \mathrm{~h}$ period. Based on the work of Irie [5], we estimate the mag- 


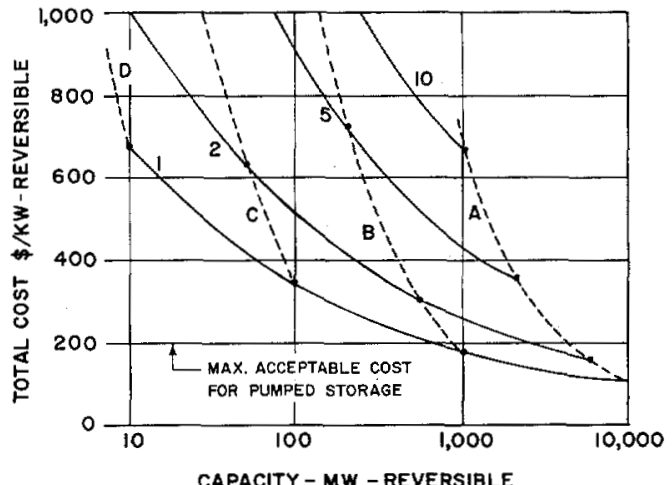

Fig. 2. Superconductive energy storage costs versus reversible capacity. Letters on dashed curves are for maximum stored energy: $A$ $10000, B-1000, C-100$, and $D-10 \mathrm{MWh}$. Numbers on solid curves are hours for complete discharge at installed reversible capacity rating. The converter equipment is assumed to cost $\$ 40 / \mathrm{kW}$, the magnet superconductor $\$ 60 / \mathrm{kg}$, and the magnet 3 times the superconductor cost.

netic hysteresis loss at 1 percent or $50 \mathrm{MWh}$ per day. As shown below we estimate mechanical hysteresis loss at $1 / 2 \mathrm{per}$. cent or $25 \mathrm{MWh}$ per day. The loss associated with leads is taken as $1 / 4$ percent, $1 \mathrm{MW}$ or $24 \mathrm{MWh}$ per day, which allows currents up to $2 \times 10^{6} \mathrm{~A}$ in total through the thermal barrier into the various modules. Thermal radiation losses through the structure and superinsulation need not exceed 1 percent, $4 \mathrm{MW}$ or $96 \mathrm{MWh}$. The total loss during a $24 \mathrm{~h}$ period is $387 \mathrm{MWh}$ for $4800 \mathrm{MWh}$ delivered, or 8 percent of the delivered energy and 4 percent of the total stored energy. In contrast, hydrostorage would deliver $4800 \mathrm{MWh}$ with a $2400 \mathrm{MWh}$ loss or 50 percent loss compared to the delivered energy.

A major unknown is the anelastic or mechanical hysteresis loss into the cryogenic region. The $1 / 2$ percent taken above was estimated as follows. Each cycle a strain energy $\sigma_{w}^{2} / 2 Y$ per unit volume is absorbed by the structure, where $Y$ is the modulus of elasticity and $\sigma_{w}$ is the working stress. This represents a fraction $\sigma_{w} / 2 Y$ of the total electromagnetic stored energy which approaches 0.1 percent if the structure has been minimized. With a refrigeration penalty of 500 watts/watt we have therefore assumed an anelasticity of $10^{-2}$, which is perhaps reasonable, in arriving at $1 / 2$ percent loss.

Additional advantages for this system are the flexibility of control and speed of response, which are helpful from a transient stability point of view $[6,7,8]$. Electromechanical oscillations can be damped effectively by proper design of the control system. Computer studies reveal that this damping capability even without superconductivity at cryogenic or ambient temperature is useful in smaller sizes when energy storage in large amounts is not the primary objective.

It is in the larger sizes in terms of reversible power capability and maximum energy storage capability that one looks for favorable economic comparisons with conventional pumped hydrostorage. Fig. 2 shows that sizes in the reversible range of from 1000 to $10000 \mathrm{MW}$ might be expected to be reduced below $\$ 200 / \mathrm{kW}$ reversible for energy storage capabilities in the 1000 to $10000 \mathrm{MWh}$ range and with a suitable daily load cycle. Note that the cost for large sizes approaches the cost for ac/dc conversion equipment and is correspondingly less sensitive to magnet costs.

\section{SUPERCONDUCTOR OPTIMIZATION}

Brooks coils, thin wall solenoids, and thin wall toroids are evaluated as to their utilization of superconductor material. The Brooks coil is a special shaped solenoid which is more like a dipole with outer diameter/inner diameter $\simeq 2$ and length/inner diameter $\simeq 1 / 2$. Such a coil will ordinarily provide the maximum inductance for a given length wire. A figure of merit $E / M$, energy stored/superconductor mass, can be established for each configuration. The figures of merit in joules/gram for the toroid, the solenoid, and the Brooks coil are $(1.35,2.38,2.38) 10^{-9}(\alpha r / \rho)$, respectively, where $\alpha$ is the superconductor pinning strength $H j$ in $\mathrm{G} \cdot \mathrm{A} / \mathrm{cm}^{2}, \rho$ is the superconductor density in $\mathrm{gram} / \mathrm{cm}^{3}$, and $r$ in $\mathrm{cm}$ is the inner radius of the solenoids and the minor radius of the toroid. In this case the major radius $R$ of the toroid is chosen equal to twice the minor radius since the figure of merit is a maximum near $R / r=2$. The solenoid was chosen to use the same amount of material as in the toroid with the same field and radius which gives a solenoid length of $2 \pi r$. The pinning strength $\alpha$ is approximately constant for limited field regions and is ty pically $10^{10}$.

It is seen that for a given amount of superconductor the solenoid will store 1.77 times the energy stored in a toroid. It appears that a Brooks coil and the above selected solenoid are equally efficient. However, for a given energy the long solenoid will have a smaller radius and therefore a lower figure of merit. Taking $10000 \mathrm{MWh}$ at 100 kilogauss as an example, $E / M$ (Brooks coil) is $10.7\left(10^{-6}\right)(\alpha / \rho)$ for $r=$ $4520 \mathrm{~cm}$, while $E / M$ (solenoid) is $8.78\left(10^{-6}\right)(\alpha / \rho)$ for $r=$ $3640 \mathrm{~cm}$. In order to limit the peak field, Brooks coils must be constructed with very low current densities; the above $10000 \mathrm{MWh}$ example uses only $39.5 \mathrm{~A} / \mathrm{cm}^{2}$.

\section{STRUCTURE AND CRYOGENIC OPTIMIZATION}

It has been shown by Levy [9] that the minimum structural mass required to hold together a magnet system is proportional to the stored energy

$$
M \geqslant \frac{\rho_{s} E_{m}}{\sigma_{w}}
$$

where $\rho_{s}$ is the structure density, $\sigma_{w}$ is the working stress, and $E_{m}$ is the total magnetic energy. If all the structural elements are in tension, the equality holds; if some of the structure is in compression, then the mass is greater than the lower limit shown in equation (2).

Taking a solenoid as an example, we assume that the winding can be treated as a solid cylinder [10]. By making the approximation that distributed magnetic forces can be replaced by an internal pressure $P=H^{2} / 8 \pi$, we obtain

$$
\frac{t}{r}=\sqrt{\frac{\sigma_{w}+P}{\sigma_{w}-P}}-1
$$

where $t$ is the winding thickness and $r$ the inner radius. In the limit of small $P / \sigma_{w}$ the mass calculated from equation 
(3) is that predicted by equation (2). As the windings become thicker, more and more unnecessary structure is used. Some representative values of the extra structure in percents are $10,15,23,32$, and 48 for $P / \sigma_{w}=0.1,0.2,0.3,0.4$, and 0.5 , respectively. The same general principle can be shown for the toroid, but not in a simple analytical form. In addition a toroid requires central structure in compression, which leads to structural inefficiencies. There is no optimum size for structural reasons; good design approaches a constant mass per energy unit independent of size. Regarding the selection of field, however, $P / \sigma_{w}$ should be as small as possible while consistent with other requirements.

Cryogenic losses through the insulation modify the above tendency towards larger structures with lower magnetic fields. The loss through $25 \mathrm{~cm}$ of superinsulation is about 7.5 $\mu \mathrm{W} / \mathrm{cm}^{2}$. Considering toroids with $r / R=1 / 2$, this loss at room temperature amounts to $0.3 r^{2}$ watts, where $r$ is in $\mathrm{cm}$. The energy stored in the toroid is $4.25\left(10^{-8}\right) r^{3} H_{m}^{2}$ joules, where $H_{m}$ is the maximum field in gauss. Setting this loss at $1 \frac{1}{2}$ percent, for example, defines a 100 kilogauss toroid with $r=4400 \mathrm{~cm}$ and $R=8800 \mathrm{~cm}$.

\section{MAGNET DESIGN}

The stress level for copper in a copper-superconductor composite conductor should be held below the 12000 psi yield point for oxygen free high conductivity copper (OFHC copper). There are at least two good reasons for this choice: 1) the mechanical hysteresis mentioned earlier would be increased as the material yields, and 2) the electrical resistance of OFHC copper continues to increase with cold work which could take place once per day as energy is cycled out and in. The stress picture can be improved by interwinding strainless steel strips similar to those used by Purcell and Desportes [10] for a bubble chamber magnet. Since Young's modulus for stainless steel is twice that for copper, one can increase the working stress up to a maximum of 24000 psi. With a 2:1 copper to steel ratio an average stress level of 16000 psi can be achieved without exceeding 12000 psi in the copper.

The superconductor filaments should be small enough so that current sharing [11] can take place and be twisted to reduce magnetization currents [12]. The copper should be subdivided into enough turns so that sufficient surface cooling for a total thermal stability is possible [13].

In general, the stress requirements are more severe than are the electrical stability requirements, although one can reduce the margins for stability by building a simpler magnet with more steel, less copper, and fewer turns. This achieves $\sigma_{w}=24000 \mathrm{psi}$ and eventually could reach the thermal stability limit as well.

\section{CONCLUSIONS}

Energy storage by superconductive inductors appears to be a realistic possibility for power systems. The toroidal configuration seems to be satisfactory because its external field is zero. Polygonally oriented solenoids might also be suitable. The optimization of superconductor material does not appear to be critical because of the overwhelming require- ments for structure. All figures of merit show clearly that large sizes. are more efficient than small sizes, that the pinning strength $\alpha$ is the important superconductor parameter, and that low field units would be equally satisfactory if the cryogenic losses could be limited. Computer studies show that by proper design of the control system, effective damping of system electromechanical oscillations can be achieved.

\section{REFERENCES}

[1] Laquer, H. L., Ribe, F. L., and Weldon, D. M., "Energy Storage and Switching with Superconductors as a Power Source for Magnetic Fields in Pulsed Thermonuclear Experiments and Reactors," Intersociety Energy Conversion Engineering Conference, Boston, Mass., Aug. 3-5, 1971.

[2] Girard, B. and Sauzade, M., "Study and Construction of a Superconducting Coil for Energy Storage," International Institute of Refrigeration, Low Temperatures and Electric Power, 1969, p. 158.

[3] Spiel, D. E. and Boom, R. W., "Superconducting Energy Storage with a Flux Pump,"ibid., p. 15.

[4] Ferrier, M., "Stockage d'Energie dans un Enroulement Supraconducteur," ibid., p. 150.

[5] Irie, F. and Yamafuji, Y., "Some Fundamental Problems of Superconducting Energy Storage," ibid., p. 39.

[6] Peterson, H. A., Phadke, A. G., and Reitan, D. K., "Transients in EHVDC Power Systems: Part I-Rectifier Fault Currents," IEEE Trans. Power Apparatus and Systems, vol. PAS-88, No. 7, pp. 981-989, July, 1969.

[7] Peterson, H. A., and Krause, P. C., "A Direct- and QuadratureAxis Representation of a Parallel AC and DC Power System," IEEE Trans. Power Apparatus and Systems, Vol. PAS-85, pp. 201-225, 1966.

[8] Peterson, H. A. and Krause, D. C., "Damping of Power Swings in a Parallel AC and DC System," IEEE Trans. Power Apparatus and Systems, Vol. PAS-85, pp. 1231-1230, 1966.

[9] Levy, R. H., "Comments on Radiation Shielding of Space Vehicles by Means of Superconducting Coils," ARS Journal, 787, June 1962.

[10] Purcell, J. R. and Desportes, H., Argonne National Laboratory, private information.

[11] Stekly, Z. J. J., Thome, R., and Strauss, B., "Principles of Stability in Cooled Superconducting Magnets," Summer Study on Superconducting Devices, Brookhaven National Laboratory, BNL 50155, p. 748, July 1968.

[12] Smith, P. F., Wilson, M. N., Walters, C. R., and Lewin, J. D., "Intrinsically Stable Conductors," ibid., p. 913.

[13] Whetstone, C. N. and Boom, R. W., "Nucleate Cooling Stability for Superconductor-Normal Metal Composite Conductors in Liquid Helium," Advances in Cryogenic Engineering, Vol. 13, p. 68, 1968.

Readout Performance Analysis of a Cryogenic Magnetooptical Data Storage System

\section{B. R. BROWN}

Abstract-The readout signal and noise generation processes have been studied for a magnetooptical data storage model using a GaAs laser source for both thermal writing and Faraday readout on a Fe doped EuO film. Experience with a laboratory model indicates that the usual analysis which treats only detector shot noise leads to incorrect values for optimum film thickness and analyzer angle. For additional details, see Digests of the 1972 Intermag Conference (available from IEEE Headquarters).

Paper 59.2, presented at the 1972 INTERMAG Conference, Kyoto, Japan, April 10-13.

The author is with the IBM Research Laboratory, San Jose, Calif. 